\title{
A SHORT PROOF FOR A.E. CONVERGENCE OF GENERALIZED CONDITIONAL EXPECTATIONS
}

\author{
D. LANDERS AND L. ROGGE
}

\begin{abstract}
Let $L_{s}(\mu)$ be the space of real valued random variables with $\mu\left(|f|^{\mid}\right)<$ $\infty, 1<s<\infty$. Let $C \subset L_{s}(\mu)$ be a closed convex set. For each $f \in L_{s}(\mu)$ there exists a unique element $\mu_{s}(f \mid C)$ with $\left\|f-\mu_{s}(f \mid C)\right\|_{s}<\|f-c\|_{s}$ for every $c \in C$. Let $C_{n}$ be a decreasing or increasing sequence of closed convex lattices converging to the closed convex lattice $C_{\infty}$. We show that $\mu_{s}\left(f \mid C_{n}\right) \rightarrow \mu_{s}\left(f \mid C_{\infty}\right) \mu$-a.e. for every $f \in L_{s}(\mu)$.

This result contains the results of a.e. convergence of prediction sequences of Ando-Amemiya and the result of Brunk and Johansen of a.e. convergence of conditional expectations given $\sigma$-lattices.
\end{abstract}

Let $\mu$ be a measure defined on a $\sigma$-algebra $Q$ over $\Omega$. For each $s$ with $1<s<\infty$ denote by $L_{s}(\mu)$ the space of equivalence classes of real valued random variables $f$ with $\mu\left(|f|^{s}\right)<\infty$. Then $L_{s}(\mu)$ is a uniformly convex Banach space. Hence for each closed convex set $C$ and each $f \in L_{s}(\mu)$ there exists a unique element $\mu_{s}(f \mid C)$ fulfilling

(i) $\mu_{s}(f \mid C) \in C$,

(ii) $\left\|f-\mu_{s}(f \mid C)\right\|_{s}<\|f-c\|_{s}$ for all $c \in C$, where $\|f\|_{s}=\left[\mu\left(|f|^{s}\right)\right]^{1 / s}$.

If $\mu$ is a probability measure $P, s=2, \mathscr{B} \subset \mathbb{Q}$ is a sub- $\sigma$-field and $C$ is the system of all square integrable equivalence classes of functions which contain a $\mathscr{B}$-measurable function, then $P_{2}(f \mid C)$ is the usual conditional expectation of $f$ given $\mathscr{B}$, i.e. $P_{2}(f \mid C)=P^{\mathscr{B}} f$.

If $\mu$ is a probability measure, $s \neq 2, \mathscr{B} \subset \mathbb{Q}$ is a sub- $\sigma$-field and $C$ is the system of all equivalence classes of functions of $L_{s}(P)$ which contain a $\mathscr{B}$-measurable function, then $P_{s}(f \mid C)$ is the $s$-prediction $P_{s}^{\$ S} f$ of Ando-Amemiya [1].

If $s=2, \mathbb{E} \subset \mathbb{Q}$ is a sub- $\sigma$-lattice and $C$ is the system of all square integrable equivalence classes of functions which contain a $\mathbb{E}$-measurable function, then $\mu_{2}(f \mid C)$ is the conditional expectation $\mu(f \mid \Subset)$ of $f$ given $\Subset$ in the sense of [2].

Let $C_{n}$ be a decreasing or increasing sequence of closed convex subsets of $L_{s}(\mu)$. Put $C_{\infty}=\bigcap_{n \in N} C_{n}$ for the decreasing case and let $C_{\infty}$ be the closure of $\cup_{n \in N} C_{n}$ with respect to \|\|$_{s}$ for the increasing case.

Using the definition (i), (ii) of $\mu_{s}(f \mid C)$ one directly obtains

$$
\left\|f-\mu_{s}\left(f \mid C_{n}\right)\right\|_{s} \rightarrow\left\|f-\mu_{s}\left(f \mid C_{\infty}\right)\right\|_{s} .
$$

Received by the editors March 27, 1979.

AMS (MOS) subject classifications (1970). Primary 60G45; Secondary 60F99.

Key words and phrases. Pointwise convergence, projections on closed convex sets, conditional expectations. 
The following theorem gives a very short proof for the $\mu$-a.e. convergence of $\mu_{s}\left(f \mid C_{n}\right)$ to $\mu_{s}\left(f \mid C_{\infty}\right)$. The proof is even for the classical conditional expectation $P^{\mathscr{B}} f$ shorter and more transparent than the known ones. It contains the a.e. convergence result given in [1] and [2].

$C$ is a lattice if $f, g \in C$ imply $f \vee g, f \wedge g \in C$ where $f \vee g[f \wedge g]$ is the pointwise maximum [minimum] of $f$ and $g$.

If $C_{n}$ is a decreasing or increasing sequence of closed convex lattices then $C_{\infty}$ is a closed convex lattice too.

THEOREM. Let $1<s<\infty$ and $C_{n} \subset L_{s}(\mu), n \in \mathbf{N}$, be an increasing or decreasing sequence of closed convex lattices converging to the closed convex lattice $C_{\infty}$. Then

$$
\mu_{s}\left(f \mid C_{n}\right) \rightarrow \mu_{s}\left(f \mid C_{\infty}\right) \quad \mu \text {-a.e. for every } f \in L_{s}(\mu) .
$$

Proof. Let $f, g, h \in L_{s}(\mu)$. Using the trivial identity

$$
\begin{gathered}
|f(\omega)-g(\omega) \wedge h(\omega)|^{s}+|f(\omega)-g(\omega) \vee h(\omega)|^{s} \\
=|f(\omega)-g(\omega)|^{s}+|f(\omega)-h(\omega)|^{s}
\end{gathered}
$$

we obtain

$$
\int|f-g \wedge h|^{s} d \mu+\int|f-g \vee h|^{s} d \mu=\int|f-g|^{s} d \mu+\int|f-h|^{s} d \mu .
$$

Let $C_{n}$ be increasing. We shall show that for $n<m$

$$
\left\|f-\mu_{s}\left(f \mid C_{n}\right) \wedge \cdots \wedge \mu_{s}\left(f \mid C_{m}\right)\right\|_{s}<\left\|f-\mu_{s}\left(f \mid C_{n}\right) \wedge \cdots \wedge \mu_{s}\left(f \mid C_{m-1}\right)\right\|_{s}
$$

We apply (1) to $g=\mu_{s}\left(f \mid C_{n}\right) \wedge \cdots \wedge \mu_{s}\left(f \mid C_{m-1}\right), h=\mu_{s}\left(f \mid C_{m}\right)$. If (2) would be false, (1) implies

$$
\begin{aligned}
\|f-g \vee h\|_{s} & =\left\|f-\left[\mu_{s}\left(f \mid C_{n}\right) \wedge \cdots \wedge \mu_{s}\left(f \mid C_{m-1}\right)\right] \vee \mu_{s}\left(f \mid C_{m}\right)\right\|_{s} \\
& <\left\|f-\mu_{s}\left(f \mid C_{m}\right)\right\|_{s}
\end{aligned}
$$

which contradicts

$$
\left[\mu_{s}\left(f \mid C_{n}\right) \wedge \cdots \wedge \mu_{s}\left(f \mid C_{m-1}\right)\right] \vee \mu_{s}\left(f \mid C_{m}\right) \in C_{m} .
$$

From (2) we obtain for all $n<m$

$$
\left\|f-\mu_{s}\left(f \mid C_{n}\right) \wedge \cdots \wedge \mu_{s}\left(f \mid C_{m}\right)\right\|_{s}<\left\|f-\mu_{s}\left(f \mid C_{n}\right)\right\|_{s}
$$

Using the lemma of Fatou $(m \rightarrow \infty)$, (3) implies that for all $n$

$$
\left\|f-\bigwedge_{m>n} \mu_{s}\left(f \mid C_{m}\right)\right\|_{s}<\left\|f-\mu_{s}\left(f \mid C_{n}\right)\right\|_{s}
$$

Applying once more the lemma of Fatou $(n \rightarrow \infty),(4)$ and (*) imply

$$
\begin{aligned}
\left\|f-\frac{\lim }{n \in \mathbf{N}} \mu_{s}\left(f \mid C_{n}\right)\right\|_{s} & <\lim _{n \in \mathbf{N}}\left\|f-\mu_{s}\left(f \mid C_{n}\right)\right\|_{s} \\
& =\left\|f-\mu_{s}\left(f \mid C_{\infty}\right)\right\|_{s} .
\end{aligned}
$$

Since $\mu_{s}\left(f \mid C_{n}\right) \wedge \cdots \wedge \mu_{s}\left(f \mid C_{m}\right) \in C_{m} \subset C_{\infty}$ and $C_{\infty}$ is \|\|$_{s}$-closed, (4) and (5) imply that

$$
\lim _{n \in \mathbf{N}} \mu_{s}\left(f \mid C_{n}\right) \in C_{\infty}
$$


Hence

$$
\lim _{n \in \mathbf{N}} \mu_{s}\left(f \mid C_{n}\right)=\mu_{s}\left(f \mid C_{\infty}\right)
$$

Using

$$
\begin{aligned}
\| f-\mu_{s}\left(f \mid C_{n}\right) & \vee \cdots \vee \mu_{s}\left(f \mid C_{m}\right) \|_{s} \\
& <\left\|f-\mu_{s}\left(f \mid C_{n}\right) \vee \cdots \vee \mu_{s}\left(f \mid C_{m-1}\right)\right\|_{s},
\end{aligned}
$$

which follows again from (1), one obtains completely analogously $\overline{\lim }_{n \in N} \mu_{s}\left(f \mid C_{n}\right)$ $=\mu_{s}\left(f \mid C_{\infty}\right)$, which yields the assertion for the increasing case. If $C_{n}$ is decreasing one obtains from (1) [instead of (2) and (2)*] for $n<m$ :

$$
\begin{gathered}
\left\|f-\mu_{s}\left(f \mid C_{n}\right) \wedge \cdots \wedge \mu_{s}\left(f \mid C_{m}\right)\right\|_{s}<\left\|f-\mu_{s}\left(f \mid C_{n+1}\right) \wedge \cdots \wedge \mu_{s}\left(f \mid C_{m}\right)\right\|_{s}, \\
\left\|f-\mu_{s}\left(f \mid C_{n}\right) \vee \cdots \vee \mu_{s}\left(f \mid C_{m}\right)\right\|_{s}<\left\|f-\mu_{s}\left(f \mid C_{n+1}\right) \vee \cdots \vee \mu_{s}\left(f \mid C_{m}\right)\right\|_{s} .
\end{gathered}
$$

From (6) we obtain the assertion completely analogously as in the increasing case.

\section{REFERENCES}

1. T. Ando and I. Amemiya, Almost everywhere convergence of prediction sequence in $L_{p}(1<p<\infty)$, Z. Wahrscheinlichkeitstheorie und Verw. Gebiete 4 (1965), 113-120.

2. R. E. Barlow, D. J. Bartholomew, J. M. Bremner and H. D. Brunk, Statistical inference under order restrictions, Wiley, New York, 1972.

Mathematisches Institut der Universität zu Köln, Weyertal 86-90, D-5000 Köln 41, Federal REPUBLIC OF GerMaNY

Fachbereich Wirtschaftswissenschaften/Statistix, Universitït Konstanz, PostFach 7733, D-7750 Konstanz, Federal Republic of Germany 